\title{
Randomised Clinical Trial to Evaluate Safety and Efficacy of Convalescent Plasma Use Among Hospitalized Patients with COVID-19 (PERUCONPLASMA): A Structured Summary af a Study Protocol for a Randomised Controlled Trial.
}

\section{Alonso Soto}

Biomedical Sciences Research Institute. School of Medicine. Universidad Ricardo Palma

\section{Fiorella Krapp}

Tropical Medicine Institute Alexander von Humboldt. Universidad Peruana Cayetano Heredia

Alex Vargas ( $\nabla$ alex.vargas.r@upch.pe )

School of Medicine. Universidad Peruana Cayetano Heredia https://orcid.org/0000-0001-9847-9432

\section{Lucía Cabrejos}

School of Medicine. Universidad Peruana Cayetano Heredia

\section{Enrique Argumanis}

INEN: Instituto Nacional de Enfermedades Neoplasicas

\section{Patricia L García}

Department of Hemotherapy and Blood Transfusion. Instituto Nacional de Salud del Niño San Borja Karina Altamirano

Department of Clinical Pathology and Pathological Anatomy. Hospital Nacional Hipolito Unanue

\section{Martín Montes}

Tropical Medicine Institute Alexander von Humboldt. Universidad Peruana Cayetano Heredia

\section{Pamela R Chacón-Uscamaita}

Emerge, Emerging Diseases and Climate Change Research Unit. School of Public Health. Universidad Peruana Cayetano Heredia

\section{Patricia J. García}

School of Public Health. Universidad Peruana Cayetano Heredia

\section{Research Article}

Keywords: COVID-19, randomised controlled trial, protocol, convalescent plasma, safety, adverse reactions, mortality. 
DOI: https://doi.org/10.21203/rs.3.rs-317097/v1

License: (c) (1) This work is licensed under a Creative Commons Attribution 4.0 International License. Read Full License 


\section{Abstract}

Objectives The general objective of this study is to test the hypothesis that administration of convalescent plasma from donors with previous diagnosis of severe COVID-19 pneumonia is safe and associated with a decrease in all-cause in-hospital mortality among hospitalized patients with COVID-19 at 30 days in comparison with standard treatment alone. The secondary objectives are: 1) to assess the efficacy of convalescent plasma to reduce the length of hospitalization; 2) to assess the efficacy of convalescent plasma to reduce the length of ICU stay; and 3) to assess the efficacy of convalescent plasma on reducing the requirement of invasive mechanical ventilation or ICU stay. Trial design PERUCONPLASMA is a llb phase open label, randomised, superiority clinical trial with 1:1 allocation taking place in real life routine clinical practice at public hospitals in Lima, Peru. Participants will be randomised to receive convalescent plasma along with local standard treatment or local standard treatment alone. After allocation all participants will be followed for a total of 30 days or until hospital discharge, whichever occurs first.

\section{Participants}

The population for the study are patients with severe disease with a confirmed laboratory test for SARSCoV-2 infection hospitalized in 3 tertiary-care hospitals in Lima-Perú.

Subjects are eligible for the trial if they meet all of the following inclusion criteria:

1. Age 18 or older

2. Hospitalization due to COVID-19 with laboratory confirmation (either with serologic, molecular or antigen test along with a compatible clinical presentation)

3. Severe or critical COVID-19 disease

Severe illness was defined by 2 or more of the following:

- Respiratory rate of 22 or more

- Hypoxemia with oxygen saturation equal or less than $93 \%$

- Abnormal blood gas analysis $(\mathrm{PaO} 2<60 \mathrm{mmHg}, \mathrm{PaCO} 2>50 \mathrm{mmHg}$ or $\mathrm{Pa} / \mathrm{FiO} 2<300)$

Critical disease was defined by either:

- Mechanical ventilation requirement less than 72 hours.

- Shock

4. Capacity to provide informed consent (patient or patient's direct relative)

5. Availability of convalescent plasma units compatible with $\mathrm{ABO}$ blood type of the subject.

Exclusion criteria: 
Subjects are not eligible for the trial if they meet any of the following criteria:

1. Contraindication for transfusion (eg, prior anaphylaxis, congestive heart failure, etc)

2. Hemodynamic instability (PA<60 $\mathrm{mmHg}$ refractory to vasopressors)

3. Uncontrolled concomitant infections\

4. Stupor or coma

5. Platelets $<50000 /$ uL or Disseminated Intravascular Coagulation

6. Serum creatinine $>3.5 \mathrm{mg} / \mathrm{dL}$ or dialysis requirement

7. Total bilirubin $>6 \mathrm{mg} / \mathrm{dL}$ or jaundice of unknown etiology

8. Myocardial infarction or acute coronary syndrome

9. Active or recent (<7 days) intracranial haemorrhage

10. Pregnancy

Donors:

The donors have to meet the following criteria: male between 30 and 60 years with a previous diagnosis of severe COVID-19 associated pneumonia within the last 3 months, with resolution of symptoms of at least 28 days. The rationale for including donors with severe disease is to maximize the probability of collecting convalescent plasma units with high tit er of neutralizing antibodies, as the technology to measure this specific type of antibodies is not routinely available in Peru. Aliquots of plasma will be stored for future quantification of neutralizing antibodies.

\section{Intervention and comparator}

Convalescent plasma from donors with previous severe COVID-19 is the investigational medical product. The experimental group will receive 1 to 2 units of 200 to $250 \mathrm{ml}$ of convalescent plasma along with local standard treatment. The control group will receive local standard treatment alone. The participants randomised to plasma will have evaluations at 6 hours and 24 hours to specifically evaluate possible post transfusion events. All the participants will be evaluated at day 3 , day 7 and day 30 after enrolment.

\section{Main Outcomes}

Safety outcome:

- Incidence of serious adverse reactions related to convalescent plasma transfusion within 24 hours after convalescent plasma administration.

Efficacy outcomes:

- Mortality from any cause during hospitalization at 30 days post randomisation. 
- Length of hospitalization at 30 days post randomisation or until hospital discharge.

- Duration of mechanical ventilation at 30 days post randomisation or until hospital discharge.

- Length of hospitalization in an intensive care unit at 30 days post randomisation or until hospital discharge.

Exploratory:

- Oxygen requirement evolution at days 3 and 7.

- Score Sequential Organ Failure Assessment (SOFA) evolution at days 3 and 7.

- Dynamics of inflammatory marker (Lymphocyte, C-reactive protein (CRP), D-Dimer, lactate dehydrogenase (LDH)) evolution at days 3 and 7.

- Proportion of patients progressing to multi-organ failure at 30 days post randomisation or until hospital discharge.

- Proportion of transfusion related adverse reactions at 30 days post randomisation or until hospital discharge.

\section{Randomisation}

Randomisation will be carried out within the electronic case report form (eCRF) in 1:1 ratio (receive plasma/control) in a randomisation process established by blocks of size 2,4 , and 6 . Allocation to the treatment arm of an individual patient will not be available to the investigators before completion of the whole randomisation process. Randomisation blocks will be performed with "ralloc", Stata's randomisation process v.16.0. Randomisation through the eCRF will be available 24 hours every day.

\section{Blinding_(masking).}

Both the participants and study staff will be aware of the allocated intervention.

Blinded statistical analysis will be performed.

\section{Numbers to be randomised (sample size).}

The sample size was calculated using the Fleiss formula with continuity correction to detect a mortality reduction from $50 \%$ to $20 \%$ between the two treatment arms with a confidence level of $95 \%$ and a power of $80 \%$. Based on this information, a total of 45 patients per arm would be needed. After adjustment for a drop-out rate of $10 \%$ after enrolment, a total of 50 patients per arm (100 patients in total) will be enrolled.

\section{Trial Status}

Current protocol version: 5.0 dated January 04, 2021.

Recruitment started on September 21, 2020 and is expected to finish by the end of March 2021. 
- Peruvian Register of Clinical Trials (REPEC) ID: PER-016-20, registered on June 27, 2020.

- Clinicaltrials.gov ID: NCT04497324, registered on August 4, 2020.

\section{Full protocol}

The full protocol is attached as an additional file, accessible from the Trials website (Additional file 1). In the interest in expediting dissemination of this material, the familiar formatting has been eliminated; this Letter serves as a summary of the key elements of the full protocol.

\section{Declarations}

\section{Ethics approval and consent to participate}

Approved by the ethics committee [Comité Nacional Transitorio de Ética en Investigación para la evaluación y supervisión ética de los ensayos clínicos de la enfermedad COVID-19] on June 27, 2020.

Approved by the National Health Institute Committee on June 27, 2020 ( $\mathrm{N}^{\circ}$ 203-2020-OGITT/INS). Identifier: PER-016-20.

\section{Informed consent procedure}

The investigator assessed the patient's availability to decide and potential consciousness impairment based on the Glasgow Coma Score. Fully conscious and oriented individuals (GCS 15) or individual's direct relative (for patients with GCS 14 or less) went through the standard procedure (informative interview with sub-investigator with written informed consent format, possibility to ask questions and adequate time to discuss with family and decide). If the individual wished to participate, he/she signed the informed consent form .

\section{Consent for publication}

Not applicable

\section{Availability of data and materials}

Collected de-identified data of the trial will be shared with other studies on the same topic upon relevant requests.

\section{Competing interests}

The authors declare that they have no competing interests. 


\section{Funding}

PERUCONPLASMA is an investigator-initiated clinical trial. This study is funded by the Embassy of Canada Fund for Local Initiatives. Trial funders have no role in study design, analysis, interpretation of data nor in writing the manuscript.

\section{Authors' contributions}

AS, FK and PJG conceived the study, wrote the original protocol draft and led the protocol development. EA, KA, PLG, and MM as clinical research specialists contributed with their expertise in specific topics and critically reviewed the first and subsequent protocol drafts. PRCh led the data management plan and the development of the electronic CRFs, with assistance from AV and LC. All authors read and approved the final manuscript.

\section{Acknowledgements}

We would like to thank Claudia Morales for her valuable administrative contribution.

\begin{tabular}{|ll|}
\hline Name of the author & e-mail \\
\hline Alonso Soto & alonso.soto@urp.edu.pe \\
\hline Fiorella Krapp & fiorella.krapp@upch.pe \\
\hline Alex Vargas & alex.vargas.r@upch.pe \\
\hline Lucia Cabrejos & lucia.cabrejos.h@upch.pe \\
\hline Enrique Argumanis & eargumanis@hotmail.com \\
\hline Patricia L Garcia & pgarcia@insnsb.gob.pe \\
\hline Karina Altamirano & priscillaaltamirano81@gmail.com \\
\hline Martin Montes & martin.montes@upch.pe \\
\hline Pamela Chacon & pamela.chacon@upch.pe \\
\hline Patricia J Garcia & patricia.garcia@upch.pe \\
\hline
\end{tabular}

\section{Supplementary Files}

This is a list of supplementary files associated with this preprint. Click to download.

- PERUCONPLASMAEthicalApproval.pdf 
- PERUCONPLASMAProtocol.pdf 